\title{
The limited storage capacity of gonadal adipose tissue directs the development of metabolic disorders in male $\mathrm{C} 57 \mathrm{BI} / 6 \mathrm{~J}$ mice
}

\author{
Lianne van Beek ${ }^{1,2}$ • Jan B. van Klinken ${ }^{1,2}$ - Amanda C. M. Pronk ${ }^{1,2}$. \\ Andrea D. van Dam ${ }^{2,3}$ • Eline Dirven ${ }^{1,2}$ • Patrick C. N. Rensen ${ }^{2,3}$. \\ Frits Koning ${ }^{4}$ Ko Willems van Dijk ${ }^{1,2,3}$ - Vanessa van Harmelen ${ }^{1,2}$
}

Received: 21 January 2015 / Accepted: 31 March 2015 / Published online: 12 May 2015

(C) The Author(s) 2015. This article is published with open access at Springerlink.com

\begin{abstract}
Aims/hypothesis White adipose tissue (WAT) consists of various depots with different adipocyte functionality and immune cell composition. Knowledge of WAT-depot-specific differences in expandability and immune cell influx during the development of obesity is limited, therefore we aimed to characterise different WAT depots during the development of obesity in mice.

Methods Gonadal WAT (gWAT), subcutaneous WAT (sWAT) and mesenteric WAT (mWAT) were isolated from male C57B1/6J mice with different body weights (approximately 25-60 g) and analysed. Linear and non-linear regression models were used to describe the extent of WAT depot expandability and immune cell composition as a function of body weight.

Results Whereas mouse sWAT and mWAT continued to expand with body weight, gWAT expanded mainly during the initial phase of body weight gain. The expansion diminished after the mice reached a body weight of around $40 \mathrm{~g}$. From this
\end{abstract}

Electronic supplementary material The online version of this article (doi:10.1007/s00125-015-3594-8) contains peer-reviewed but unedited supplementary material, which is available to authorised users.

Vanessa van Harmelen

V.J.A.van_Harmelen@lumc.nl

1 Department of Human Genetics, Leiden University Medical Center, Einthovenweg 20, PO Box 9600, 2300 RC Leiden, the Netherlands

2 Einthoven Laboratory for Experimental Vascular Medicine, Leiden University Medical Center, Leiden, the Netherlands

3 Department of Medicine, Division of Endocrinology, Leiden University Medical Center, Leiden, the Netherlands

4 Department of Immunohematology and Blood Transfusion, Leiden University Medical Center, Leiden, the Netherlands point on, gWAT crown-like structure formation, liver steatosis and insulin resistance occurred. Mouse WAT depots showed major differences in immune cell composition: gWAT consisted mainly of macrophages, whereas sWAT and mWAT primarily contained lymphocytes.

Conclusions/interpretation Marked inter-depot differences exist in WAT immune cell composition and expandability. The limited storage capacity of gWAT seems to direct the development of metabolic disorders in male $\mathrm{C} 57 \mathrm{Bl} / 6 \mathrm{~J}$ mice.

Keywords Adipocyte size - Crown-like structure · Inflammation · Obesity · Visceral · White adipose tissue

$\begin{array}{ll}\text { Abbreviations } \\ \text { BAT } & \text { Brown adipose tissue } \\ \text { CLS } & \text { Crown-like structures } \\ \text { gWAT } & \text { Gonadal white adipose tissue } \\ \text { HFD } & \text { High-fat diet } \\ \text { IR } & \text { Insulin resistance } \\ \text { mWAT } & \text { Mesenteric white adipose tissue } \\ \text { SVF } & \text { Stromal vascular fraction } \\ \text { sWAT } & \text { Subcutaneous white adipose tissue } \\ \text { TG } & \text { Triacylglycerol } \\ \text { WAT } & \text { White adipose tissue }\end{array}$

\section{Introduction}

White adipose tissue (WAT) is the main energy storage organ, and is distributed over various depots. The regional distribution and inflammatory status of WAT are strongly associated with the development of metabolic disorders. Excessive abdominal fat, or central obesity, is known to be a strong risk factor for type 2 diabetes 
mellitus and cardiovascular disease $[1,2]$. WAT can be divided into subcutaneous WAT (sWAT) and visceral WAT (vWAT), located underneath the skin and around the abdominal organs, respectively. Mouse vWAT is generally subdivided into mesenteric WAT (mWAT; between the organs) and gonadal WAT (gWAT; around the testes). While WAT was originally considered an organ with homogeneous function, vWAT is now thought to exert more adverse effects on health compared with sWAT [2-4]. These pathophysiological differences in WAT depots are linked to the metabolic and inflammatory status of the tissue.

Due to excessive fat accumulation in WAT during obesity, adipocytes become stressed and release increased amounts of fatty acids and pro-inflammatory adipokines and chemokines. These inflammatory signals induce immune cell infiltration and dysfunction of the obese WAT [5-7]. Macrophage accumulation, or more specifically the presence of crown-like structures (CLS), in the WAT is associated with adipocyte death caused by cellular lipid overload [8,9]. Furthermore, $\mathrm{T}$ and $\mathrm{B}$ lymphocytes are increased in WAT during obesity and contribute to the development of metabolic disorders $[10,11]$. Pro-inflammatory cytokines produced by both adipocytes and infiltrating immune cells directly interfere with the insulin signalling pathway, thereby affecting insulin sensitivity both locally and systemically, leading to insulin resistance (IR) and type 2 diabetes $[12,13]$. Compared with sWAT, vWAT secretes more fatty acids and pro-inflammatory cytokines and has a higher infiltration of cytotoxic $\mathrm{T}$ cells and macrophages during obesity [14-16].

Most of the human studies on WAT inflammation compare WAT between lean and obese individuals and consider only one WAT depot. The majority of mouse studies use male $\mathrm{C} 57 \mathrm{~B} 1 / 6 \mathrm{~J}$ mice as a model for obesity induced by a high-fat diet (HFD) and assess only gWAT, whereas sWAT and mWAT are neglected [17]. As different WAT depots have different functions and cellular composition, it is of importance to determine the functional and immunological phenotypes of the various WAT depots. Moreover, longitudinal studies following the development of obesity are sparse and, as a consequence, the inflammatory response of the different WAT depots during body weight gain has, until now, been poorly characterised. Therefore, the aim of the current study was to phenotype the different WAT depots and to determine regional differences with regard to WAT expandability and inflammation in male $\mathrm{C} 57 \mathrm{Bl} / 6 \mathrm{~J}$ mice during the development of HFD-induced obesity. In addition, we set out to develop a set of linear and non-linear regression models to describe organ weights and WAT (immune cell) composition as a function of body weight.

\section{Methods}

Animals Experiments were performed with six different batches of male $\mathrm{C} 57 \mathrm{Bl} / 6 \mathrm{~J}$ mice (Charles River, Maastricht, the Netherlands). The batches differed in duration (434 weeks) and type of HFD (45\% or $60 \%$ energy derived from lard fat; D12451 or D12492, Research Diet Services, Wijk bij Duurstede, the Netherlands) (electronic supplementary material [ESM] Table 1). Body weight was measured and lean and fat mass was assessed by MRI-based body composition analysis (Echo MRI, Echo Medical Systems, Houston, TX, USA). At the end of the diet intervention, mice were killed, perfused and organs were dissected for further analysis. All experiments were approved by the animal ethics committee of Leiden University Medical Center.

Adipocyte and stromal vascular cell isolation Depots of gWAT (one side), sWAT (posterior, one side) and mWAT were dissected from the mice and kept in PBS after the diet intervention. Tissues were processed for adipocyte size determination as previously described [18]. Adipocyte number per fat pad was calculated from the fat pad mass and adipocyte size. The residue of the WAT filtrate was used for the isolation of stromal vascular fraction (SVF) to analyse immune cell composition using flow cytometry. After centrifugation $(350 \mathrm{~g}$, $10 \mathrm{~min}$ ) the supernatant fraction was discarded and the pellet was treated with erythrocyte lysis buffer, after which the cells were counted using an automated cell counter (TC10, BioRad, Berkeley, CA, USA). The SVF was fixed using 0.5\% (vol./vol.) paraformaldehyde, stored in FACS buffer (PBS, $0.02 \%$ (vol./vol.) sodium azide, $0.5 \%$ (vol./vol.) FCS) in the dark at $4^{\circ} \mathrm{C}$ and analysed within 1 week.

Additional analyses Plasma, liver triacylglycerol (TG), adipocyte lipolysis, histology and flow cytometry analysis were performed as described in ESM Methods.

Statistics Data are presented as single data points or mean \pm SD. Statistical differences between groups were calculated with the Student's $t$ test using GraphPad Prism version 6 (GraphPad software, San Diego, CA, USA). Correlation analyses were performed by making correlation plots of body weight vs the variables measured in this study. We modelled the association between each variable and body weight using regression assuming either a linear $(y=b \times x+c ; a=1)$ or nonlinear power function. The non-linear power functions could either have $y$-intercept $\left(y=b \times x^{\mathrm{a}}+c ; a>1\right.$, exponential form), or $x$-intercept $\left(y=b \times(x-c)^{\text {a }} ; a<1\right.$, curve tapering off); $x=$ body weight, $y=$ lean, fat or individual organ mass. For each analysis the $p$ value zero slope $(p)$ indicated if the slope was significantly different from $0(b=0$, horizontal line). In addition, superiority of the non-linear (power) function over the linear model was determined by testing the hypothesis $a=1$ using 
the extra sum-of-squares $F$ test; the corresponding $p$ value was termed $p$ value linear indicated by $p^{\text {lin }}$. A Spearman rank correlation coefficient $(r)$ was determined for every association. A value of $p<0.05$ was considered statistically significant, $p$ values were corrected by Bonferroni multiple test correction when indicated; significant values in the tables are identified by $* p<0.05, * * p<0.01, * * * p<0.001$.

\section{Results}

HFD-induced changes in body composition and organ weight In order to study mice with a broad range of body weights, ranging from lean to severely obese (26.3-59.3 g, $n=54), \mathrm{C} 57 \mathrm{Bl} / 6 \mathrm{~J}$ mice were subjected to different diet interventions for a different number of weeks and with a varying fat percentage in the diet. Lean mice $(<30 \mathrm{~g})$ consisted of about $80 \%$ lean mass and $20 \%$ fat mass. Mice exposed to HFD increased both lean and fat mass, though fat mass increased relatively more (Fig. 1a, b; ESM Table 2). Obese mice consisted of up to $50 \%$ fat mass. Figure $1 \mathrm{c}$ shows that the liver weight increased non-linearly with a power $>1$ when correlated with body weight, with a substantially increased liver weight from approximately $40 \mathrm{~g}$ upwards. This was mainly caused by an increase of fat in the liver (ESM Fig. 1a). Also, heart weight had a non-linear correlation with body weight (Fig. 1d). Spleen weight correlated linearly with body weight (Fig. 1e), as did brown adipose tissue (BAT), which showed a very strong linear positive correlation with body weight (Fig. 1f). BAT lipid droplet content correlated positively both with body weight (ESM Fig. 1b) and BAT weight ( $r=0.64, p=0.0001$, data not shown).

ESM Table 2 shows the equations of the curves of the correlations between the individual organs and body weight after best-fit comparison statistics. Plasma glucose and insulin as well as plasma lipid levels were measured and correlated with body weight. Glucose increased at the start of body weight gain, after which it tapered off (Fig. 2a). As glucose levels are regulated by insulin, the flattening of the glucose curve can be linked to increasing insulin levels (Fig. 2b). Plasma total cholesterol correlated positively with body weight ( $r=0.72, p<0.0001)$, whereas other plasma lipids (TG and NEFA) did not correlate with body weight (data not shown).
Fig. 1 Correlation of HFDinduced changes in body composition and organ weight with body weight. Correlations with body weight in male C57B1/ $6 \mathrm{~J}$ mice (ranging from approximately 25 to $60 \mathrm{~g}$ ) are shown for lean mass (a), fat mass (b), and different organ weights: liver (c), heart (d), spleen (e) and interscapular BAT (f).

Associations were modelled using either a linear model or nonlinear function, $95 \%$ CIs are shown as grey bands. See ESM Table 2 for equations, correlations and $p$ values a

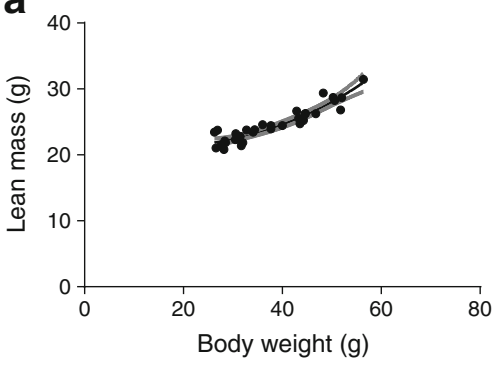

C
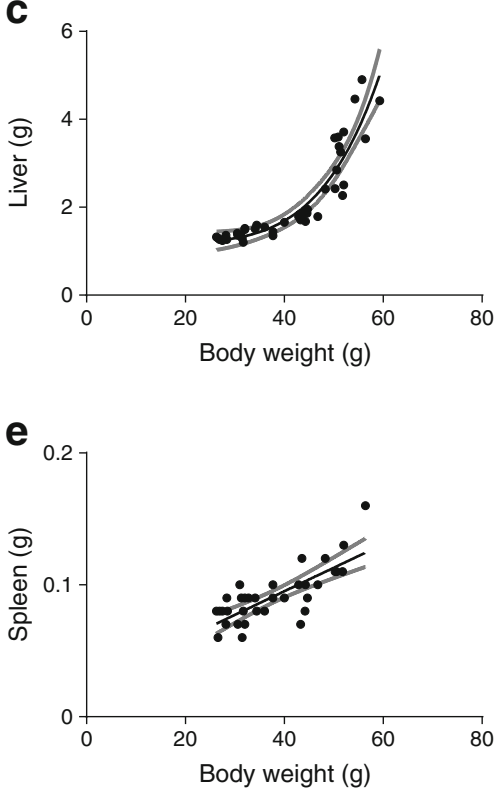

b

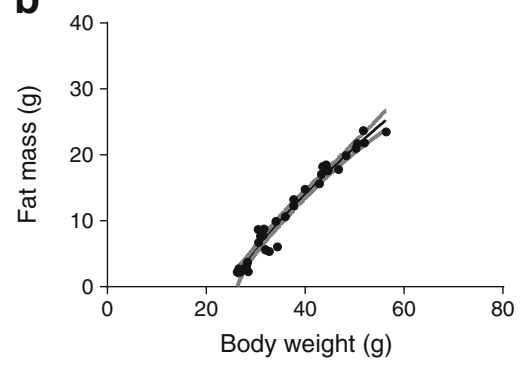

d

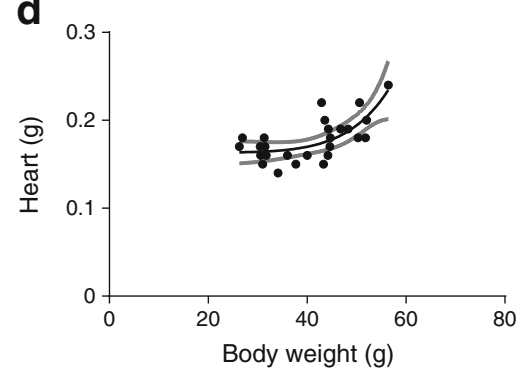

f

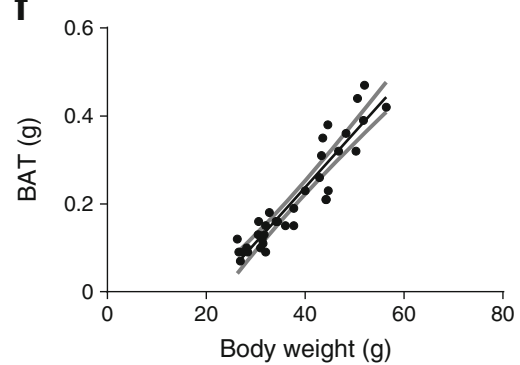




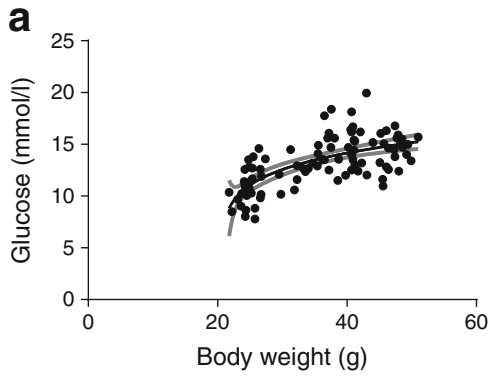

Fig. 2 Correlation of HFD-induced changes in plasma glucose and insulin levels with body weight. The correlation between body weight and plasma glucose $\left(\mathbf{a}, r=0.67, p<0.0001, p^{\text {lin }}=0.0044\right)$ and insulin $(\mathbf{b}$, $\left.r=0.93, p<0.0001, p^{\text {lin }}<0.0001\right)$ levels in male C57Bl/6J mice.

Expandability of mouse WAT depots Fat pad weight of the various WAT depots reflects expandability during HFD exposure. The gWAT depot expanded mostly during the initial phase of weight gain compared with both sWAT and mWAT. With progressing weight gain, the gWAT growth curve tapered off, whereas sWAT and mWAT continued to grow with increasing body weight (Fig. 3a, c, e; ESM Table 2). This is also illustrated by ESM Fig. 2, which shows the results when mice were divided into groups based on body weight to determine the adipocyte size distribution. Whereas for both sWAT and mWAT the adipocyte size distribution curve shifted towards larger adipocytes with higher body weight, gWAT

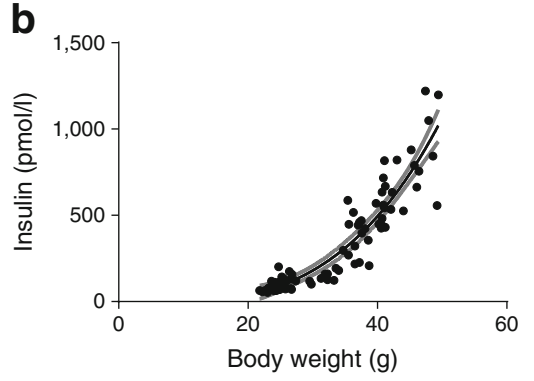

Associations were modelled using either a linear model or non-linear function; 95\% CIs are shown as grey bands. A significant $p$ value provides evidence of a non-zero slope in the linear model; a significant $p^{\text {lin }}$ value provides evidence that the association is non-linear

adipocytes remained comparable in size from approximately $40 \mathrm{~g}$ onwards. Interestingly, the gonadal adipocytes were larger compared with adipocytes of sWAT and mWAT for both lean and obese mice (Fig. 3b, d, f; ESM Table 3, 4). The potency of insulin to inhibit lipolysis in gonadal adipocytes was tested ex vivo and revealed a negative correlation with body weight (Fig. 4a), as well as with adipocyte size ( $r=-0.42, p=0.0043$, data not shown).

WAT growth is accomplished by hypertrophy (increase in size) or hyperplasia (increase in number) of the adipocytes. For all three WAT depots, there was a significant correlation between body weight and adipocyte size (Fig. 3b, d, f;
Fig. 3 Correlation of HFDinduced changes in fat pad weight and adipocyte size with body weight. Fat pad weight (a, c, e) and adipocyte size $(\mathbf{b}, \mathbf{d}, \mathbf{f})$ of gWAT, sWAT and mWAT in relation to body weight in male C57Bl/6J mice. For gWAT and sWAT, one fat pad is representative. Associations were modelled using either a linear model or non-linear function; 95\% CIs are shown as grey bands. See Table 1 and ESM Table 2 for equations, correlations and $p$ values
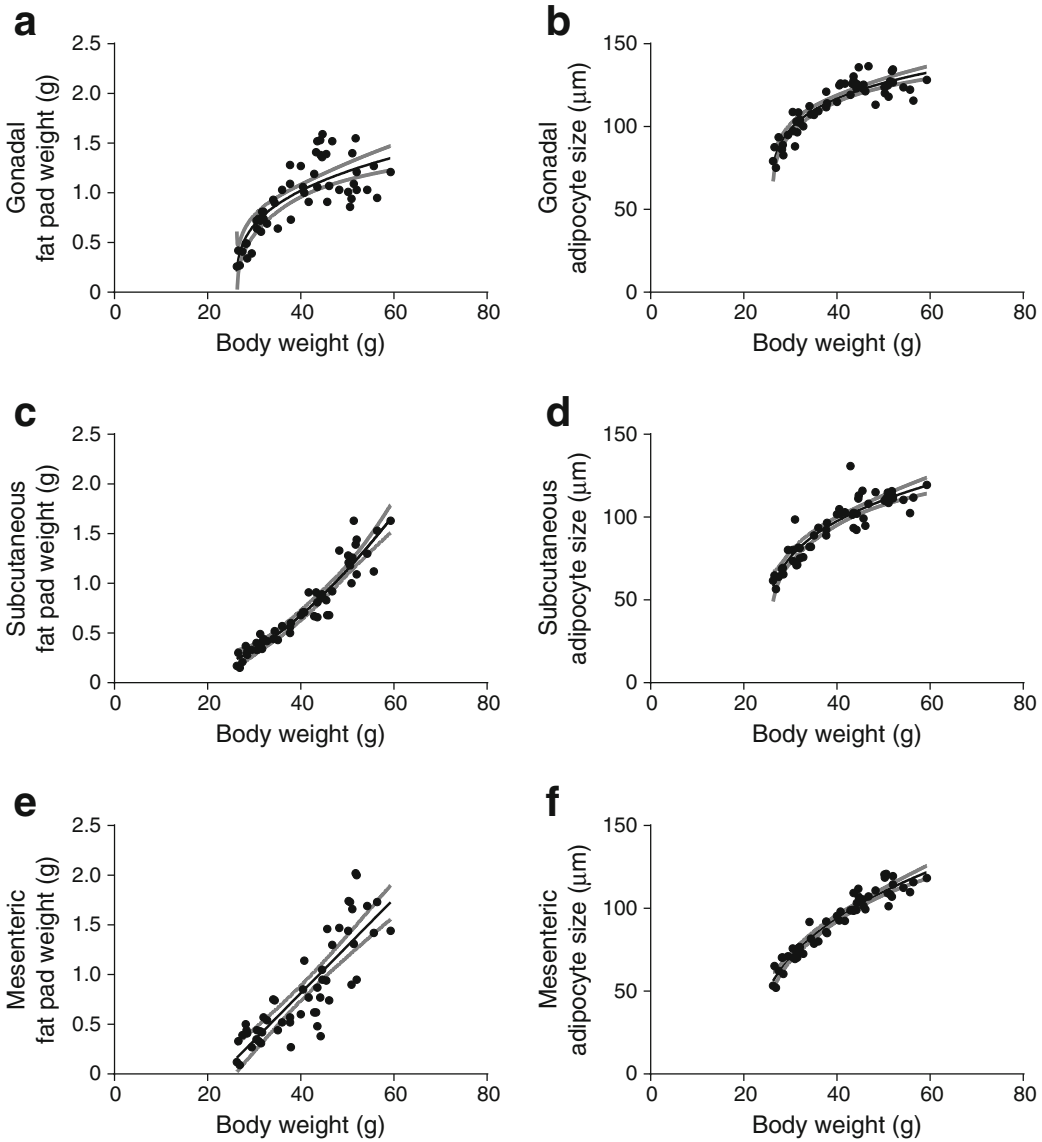

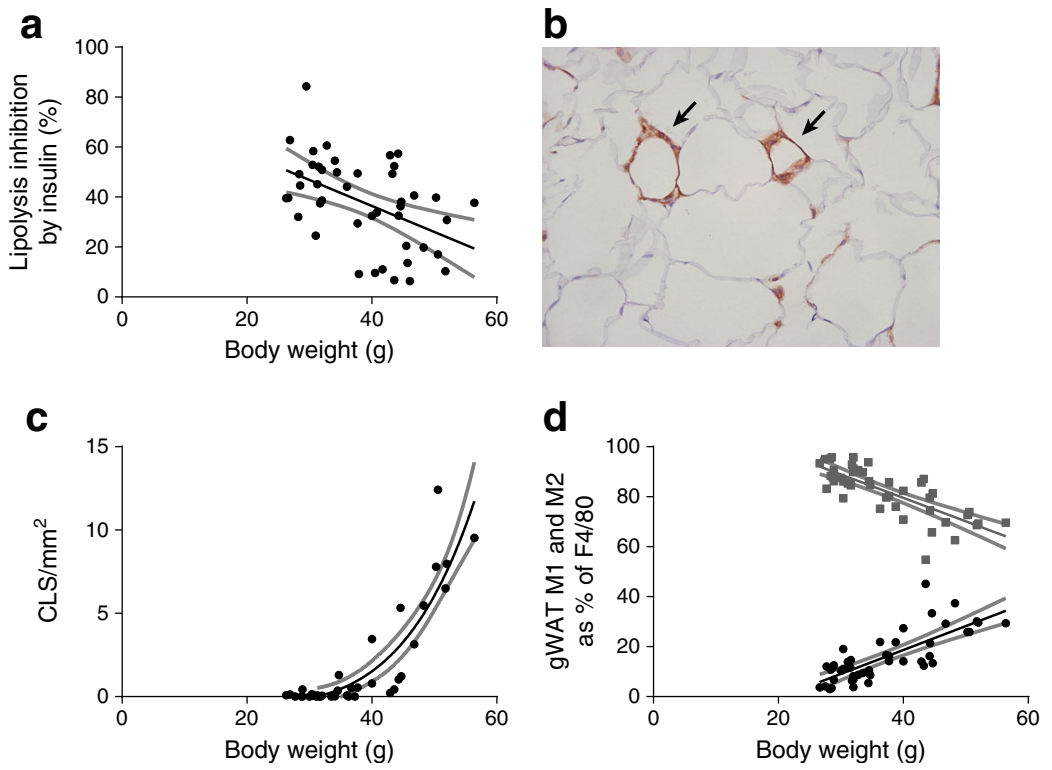

Fig. 4 Correlation of insulin responsiveness of adipocytes and macrophage phenotype in gWAT with body weight. Percentage inhibition of lipolysis by insulin of gonadal adipocytes correlated with body weight (a, $r=-0.47, p=0.0010$ ). Insulin responsiveness of the adipocytes was determined by measuring the response of the adipocytes to 8-bromo-cAMPstimulated lipolysis and the percentage inhibition thereof by insulin. F4/ 80 -stained macrophages in gWAT (b), a $\times 20$ magnification is used, CLS are indicated by arrows. CLS counts $/ \mathrm{mm}^{2}$ WAT correlated with body weight of male C57Bl/ $6 \mathrm{~J}$ mice $\left(\mathbf{c}, r=0.71, p<0.0001, p^{\text {lin }}<0.0001\right)$.

Table 1), whereas adipocyte number did not correlate with body weight (Table 1). However, when correlated with WAT depot weight, adipocyte number did show a slight positive correlation for gWAT and mWAT (data not shown). These data indicate that WAT expansion occurred predominantly by adipocyte hypertrophy and somewhat by hyperplasia in gWAT and mWAT, whereas sWAT expanded only by adipocyte hypertrophy.

HFD-induced changes in immune cell composition in mouse WAT depots WAT depots were processed to isolate the SVF, which contains immune cells as well as preadipocytes and endothelial cells. The absolute SVF cell number was determined and represented per fat pad. The SVF cell count of gWAT correlated positively with body weight, whereas the SVF cell counts of sWAT and mWAT did not correlate with body weight (Table 1). Absolute leucocyte numbers $\left(\mathrm{CD} 45^{+}\right.$cells) per fat pad showed a linear correlation with body weight for both gWAT and sWAT, whereas leucocyte numbers in mWAT showed no correlation with body weight (ESM Figs 3a, 4a, 5a). Absolute T cell numbers in gWAT and sWAT, but not mWAT, correlated positively with body weight (ESM Figs 3b, 4b, 5b). Within the $\mathrm{T}$ cell population, the ratio between $\mathrm{T}$ helper cells and cytotoxic $\mathrm{T}$ cells $\left(\mathrm{CD}^{+}\right.$and $\mathrm{CD} 8^{+}$cells, respectively) was determined. In both gWAT and sWAT the

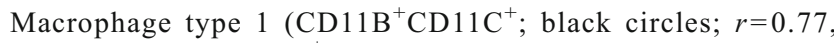

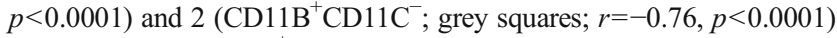
as percentage of $\mathrm{F} 4 / 80^{+}$cells in SVF of gWAT by flow cytometry (d) correlated with body weight of male $\mathrm{C} 57 \mathrm{Bl} / 6 \mathrm{~J}$ mice. Associations were modelled using either a linear or non-linear function; $95 \%$ CIs are shown as grey bands. A significant value of $p$ provides evidence of a non-zero slope in the linear model; a significant value of $p^{\text {lin }}$ provides evidence that the association is non-linear

CD4:CD8 ratio showed a negative correlation with bodyweight, which indicates a larger relative increase in cytotoxic $\mathrm{T}$ cells compared with $\mathrm{T}$ helper cells (Table 1). Absolute B cell numbers $\left(\mathrm{CD} 19^{+}\right.$cells) showed a positive correlation with body weight for gWAT (ESM Fig. 3d).

Absolute macrophage numbers ( $\mathrm{F} 4 / 80^{+}$cells) of all three WAT depots correlated positively with body weight (ESM Figs $3 \mathrm{c}, 4 \mathrm{c}, 5 \mathrm{c})$. Interestingly, absolute macrophage numbers in gWAT and sWAT showed a non-linear correlation with body weight with a power $>1$, while the correlation in mWAT was linear. WAT macrophages can form CLS, which is shown in Fig. $4 \mathrm{~b}$ by F4/80 staining of gWAT. CLS increased non-linearly with increasing body weight with a power $>1$ in the gWAT depot (Fig. 4c). Within the F4/80 $0^{+}$cell population, $\mathrm{M} 1$ and M2 macrophages were distinguished using CD11B and $\mathrm{CD} 11 \mathrm{C}$ markers $\left(\mathrm{M} 1, \mathrm{CD} 11 \mathrm{~B}^{+} \mathrm{CD} 11 \mathrm{C}^{+}\right.$; $\mathrm{M} 2$,

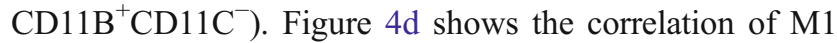
and M2 macrophages as percentages of F4/80 cells from the gWAT depot (representative for the other two depots, data not shown) with body weight. Within all adipose tissue depots, M1 macrophages were positively correlated and M2 macrophages were negatively correlated with body weight. The M1:M2 ratio also showed a strong positive correlation with body weight within all WAT depots (Table 1). This indicates relatively more M1 macrophages in WAT with a higher body 
Table 1 WAT depot composition correlated with body weight of mice on an HFD

\begin{tabular}{|c|c|c|c|c|c|c|}
\hline \multirow[t]{2}{*}{ Variable } & \multicolumn{3}{|c|}{ Correlation with body weight } & \multicolumn{3}{|l|}{ Statistics } \\
\hline & $a$ & $b$ & $c$ & $r$ & $\begin{array}{l}p \text { value }^{\mathrm{a}} \\
\text { Zero slope }\end{array}$ & $\begin{array}{l}p \text { value }^{\mathrm{a}} \\
\text { Linear }\end{array}$ \\
\hline \multicolumn{7}{|l|}{ gWAT } \\
\hline $\begin{array}{l}\text { Adipocyte size } \\
(\mu \mathrm{m} ; n=54)\end{array}$ & 0.1482 & 78.63 & 25.46 & 0.8112 & $1.40 \times 10^{-19 * * *}$ & $2.92 \times 10^{-07 * * *}$ \\
\hline $\begin{array}{l}\text { Adipocyte no./fat pad } \\
(n=46)\end{array}$ & & & & 0.1822 & 1.000 & - \\
\hline $\begin{array}{l}\text { SVF no. } / \text { /fat pad } \\
(n=54)\end{array}$ & 1 & 40,829 & $-672,214$ & 0.6768 & $1.20 \times 10^{-05 * * *}$ & 1.000 \\
\hline $\begin{array}{l}\text { Leucocytes } \\
(\% \text { CD } 45 \text { of SVF; } n=44)\end{array}$ & 1 & 0.5060 & 42.98 & 0.4131 & 0.1197 & - \\
\hline $\begin{array}{l}\text { T lymphocytes } \\
(\% \text { CD3 of SVF; } n=44)\end{array}$ & 1 & 0.2878 & 0.1631 & 0.3518 & $0.0315^{*}$ & 1.000 \\
\hline $\begin{array}{l}\text { T lymphocyte ratio } \\
(\mathrm{CD} 4: \mathrm{CD} 8 ; n=51)\end{array}$ & 1 & -0.1689 & 10.71 & -0.6287 & $7.28 \times 10^{-05 * * *}$ & 0.8226 \\
\hline $\begin{array}{l}\text { B lymphocytes } \\
\text { (\% CD19 of SVF; } n=35)\end{array}$ & 1 & 0.1398 & -1.206 & 0.3631 & 0.0963 & - \\
\hline $\begin{array}{l}\text { Macrophages } \\
(\% \mathrm{~F} 4 / 80 \text { of SVF; } n=50)\end{array}$ & & & & -0.1962 & 1.000 & - \\
\hline $\begin{array}{l}\text { Macrophage ratio } \\
(\mathrm{M} 1: \mathrm{M} 2 ; n=45)\end{array}$ & 1 & 0.01534 & -0.3574 & 0.7710 & $7.92 \times 10^{-08 * * *}$ & 1.000 \\
\hline \multicolumn{7}{|l|}{ sWAT } \\
\hline $\begin{array}{l}\text { Adipocyte size } \\
(\mu \mathrm{m} ; n=54)\end{array}$ & 0.2473 & 49.44 & 24.39 & 0.8886 & $3.26 \times 10^{-20 * * *}$ & $4.33 \times 10^{-04 * * *}$ \\
\hline $\begin{array}{l}\text { Adipocyte no./fat pad } \\
(n=46)\end{array}$ & & & & -0.03627 & 1.000 & - \\
\hline $\begin{array}{l}\text { SVF no. }{ }^{\mathrm{a}} / \mathrm{fat} \mathrm{pad} \\
(n=54)\end{array}$ & & & & 0.2907 & 1.000 & - \\
\hline $\begin{array}{l}\text { Leucocytes } \\
(\% \text { CD } 45 \text { of SVF; } n=50)\end{array}$ & 1 & 0.6146 & 32.41 & 0.4680 & $0.0036^{* *}$ & 1.000 \\
\hline $\begin{array}{l}\text { T lymphocytes } \\
(\% \text { CD } 3 \text { of SVF; } n=40)\end{array}$ & & & & 0.1068 & 1.000 & - \\
\hline $\begin{array}{l}\text { T lymphocyte ratio } \\
\text { (CD4:CD8; } n=50)\end{array}$ & 1 & -0.03013 & 2.784 & -0.4064 & 0.1818 & - \\
\hline $\begin{array}{l}\text { B lymphocytes } \\
(\% \text { CD19 of SVF; } n=43)\end{array}$ & & & & -0.07501 & 1.000 & - \\
\hline $\begin{array}{l}\text { Macrophages } \\
(\% \mathrm{~F} 4 / 80 \text { of SVF; } n=29)\end{array}$ & & & & -0.1822 & 1.000 & - \\
\hline $\begin{array}{l}\text { Macrophage ratio } \\
(\mathrm{M} 1: \mathrm{M} 2 ; n=34)\end{array}$ & 1 & 0.01025 & -0.2255 & 0.6262 & $1.95 \times 10^{-06 * * *}$ & 0.5508 \\
\hline \multicolumn{7}{|l|}{ mWAT } \\
\hline $\begin{array}{l}\text { Adipocyte size } \\
(\mu \mathrm{m} ; n=54)\end{array}$ & 0.3524 & 34.09 & 22.17 & 0.9528 & $3.17 \times 10^{-29 * * *}$ & $3.23 \times 10^{-04 * * *}$ \\
\hline $\begin{array}{l}\text { Adipocyte no./fat pad } \\
(n=46)\end{array}$ & & & & -0.06658 & 1.000 & - \\
\hline $\begin{array}{l}\text { SVF no. }{ }^{\mathrm{a}} / \mathrm{fat} \mathrm{pad} \\
(n=54)\end{array}$ & & & & 0.1914 & 1.000 & - \\
\hline $\begin{array}{l}\text { Leucocytes } \\
(\% \text { CD } 45 \text { of SVF; } n=49)\end{array}$ & 1 & -0.6190 & 97.37 & -0.3613 & 0.0729 & - \\
\hline $\begin{array}{l}\text { T lymphocytes } \\
(\% \text { CD } 3 \text { of SVF; } n=48)\end{array}$ & 1 & -0.7400 & 54.93 & -0.6355 & $7.07 \times 10^{-06 * * *}$ & 0.5445 \\
\hline $\begin{array}{l}\text { T lymphocyte ratio } \\
(\mathrm{CD} 4: \mathrm{CD} 8 ; n=48)\end{array}$ & & & & 0.06193 & 1.000 & - \\
\hline $\begin{array}{l}\text { B lymphocytes } \\
(\% \text { CD19 of SVF; } n=30)\end{array}$ & 1 & -0.9100 & 72.53 & -0.3696 & 0.0261 & - \\
\hline $\begin{array}{l}\text { Macrophages } \\
(\% \mathrm{~F} 4 / 80 \text { of SVF; } n=23)\end{array}$ & 1 & 0.9423 & -22.89 & 0.5198 & $0.0153^{*}$ & 0.1161 \\
\hline $\begin{array}{l}\text { Macrophage ratio } \\
(\mathrm{M} 1: \mathrm{M} 2 ; n=29)\end{array}$ & 1 & 0.01035 & -0.2701 & 0.6768 & $6.93 \times 10^{-04 * * *}$ & 0.2763 \\
\hline
\end{tabular}

${ }^{\text {a }} p$ value after Bonferroni multiple test correction; ${ }^{*} p<0.05, * * p<0.01,{ }^{* * *} p<0.001$

${ }^{b}$ Absolute number of cells in the SVF

No. number 
weight. Thus, HFD induces immune cell compositional changes in all three WAT depots, with an increase in immune cell numbers mainly in gWAT and sWAT.

Immune cell composition of distinct WAT depots from lean and obese mice The gWAT, sWAT and mWAT depots from either lean or obese mice (mean body weight $31.0 \pm 2.9 \mathrm{~g}$, $n=10$ and $50.1 \pm 3.6 \mathrm{~g}, n=8$, respectively) were analysed and compared with each other to determine differences in immune cell composition between the adipose tissue regions. In lean mice, approximately $60 \%$ of the SVF from gWAT and sWAT consisted of leucocytes $(57.5 \pm 9.9 \%$ and $62.7 \pm 6.2 \%$, respectively), and in mWAT this percentage was even higher ( $78.3 \pm$ $12.1 \%$ ) (ESM Table 3). In obese mice, there were no differences in leucocyte percentage in the SVF between the different WAT depots (approximately 65\%, ESM Table 4). T cells were present in all three WAT depots. Interestingly, in mWAT from lean mice the percentage of T cells in the SVF was significantly higher compared with gWAT and sWAT (ESM Table 3). The CD4:CD8 ratio in lean sWAT and mWAT was between 1 and 2, which indicates slightly more CD4 cells than CD8 cells. However, lean gWAT contained even more CD4 than CD8 cells as the ratio was around 5 (ESM Table 3). In obese mice, there were no differences in $\mathrm{T}$ cell percentages between the WAT depots (ESM Table 4). There were major differences in $\mathrm{B}$ cell content between the depots, ranging from hardly any B cells in gWAT to approximately $35 \%$ of the SVF in mWAT of lean mice and approximately $20 \%$ of the SVF in mWAT of obese mice (ESM Tables 3 and 4). The gWAT predominantly contained macrophages (approximately $30 \%$ of SVF both in lean and obese mice), while less than $10 \%$ of the SVF from sWAT consisted of macrophages in both lean and obese mice. These data indicate that there are large differences in immune cell composition between different WAT depots from both lean and obese mice.

\section{Discussion}

In the current study we determined intra-depot differences in WAT immune cell composition in relation to WAT expandability. Mouse WAT depots showed major differences in expandability and immune cell infiltration during the development of obesity. Furthermore, a body weight of approximately $40 \mathrm{~g}$ emerged as a critical tipping point from whereon metabolic dysfunction occurred, at least in male $\mathrm{C} 57 \mathrm{Bl} / 6 \mathrm{~J}$ mice.

It has been extensively shown that distinct WAT depots from both mice and humans have different metabolic functions. This is due to intrinsic differences in adipocyte characteristics but has also been attributed to differences in immune cell composition in the various depots [19-21]. Here, we have performed a direct comparison of the different WAT depots in male $\mathrm{C} 57 \mathrm{~B} 1 / 6 \mathrm{~J}$ mice and focussed simultaneously on the expandability and immune cell composition during the development of obesity, which has until now been poorly characterised. Our data confirm great variability in immune cell composition between WAT depots. The characteristics of the different mouse WAT depots already differed in the lean state, and each depot responded differently to body weight gain with respect to immune cell composition as well as expandability. Mouse gWAT expanded mostly during the initial phase of body weight gain, and increased less after a body weight of around $40 \mathrm{~g}$. Although sWAT and mWAT did not primarily expand as fast as gWAT, they both kept expanding with body weight after $40 \mathrm{~g}$. This implies that gWAT is the primary storage depot that grows initially in HFD-induced obesity, followed by sWAT and mWAT. This is also reflected by the larger gWAT adipocytes seen during both the lean and obese states, and which have also been identified by Sackmann-Sala et al, albeit only in lean mice [22].

At around a body weight of $40 \mathrm{~g}$, at which point the gWAT growth curve tapered off, the liver started to grow significantly, mainly caused by an increase in fat content. Apparently, the gWAT adipocytes were saturated and could not grow any larger to store additional fat. As a consequence, the excess fat that could not be stored in the gWAT depot was stored ectopically in the liver [23]. Moreover, around this point of body weight gain, the number of CLS started to increase in the gWAT. As CLS are found around stressed and dying adipocytes [9], the increase in numbers of CLS around $40 \mathrm{~g}$ body weight appear to be associated with increased adipocyte death. Also, insulin levels increased substantially from this point on, indicating the development of insulin resistance. Our data therefore imply that approximately $40 \mathrm{~g}$ body weight is an important tipping point in male $\mathrm{C} 57 \mathrm{Bl} / 6 \mathrm{~J}$ mice from whereon WAT and systemic metabolic dysfunction occur concomitantly. In this study, data have been exclusively obtained from male $\mathrm{C} 57 \mathrm{Bl} / 6 \mathrm{~J}$ mice in combination with HFD to induce obesity. Whether females, other mouse strains/models or humans also have such a threshold BMI at which WAT inflammation and metabolic dysfunction rapidly increase remains to be investigated.

Our data are in agreement with the study of Strissel et al, which also showed that at a certain body weight in mice gWAT stops expanding because of increased adipocyte death, whereas liver starts accumulating fat [24]. Strikingly, while our data showed a constant gWAT weight and continuous increase in CLS, they showed a reduction in both gWAT weight and CLS formation after reaching a body weight of $40 \mathrm{~g}$. This was accompanied by a reduction in adipocyte size and increased adipocyte numbers, which they attributed to newly differentiated adipocytes. One explanation for their findings might be that they fed their mice a $60 \%$ HFD for 20 weeks, which may be a more extreme intervention than those used in our study. There are several other studies 
elucidating regional differences in WAT growth [25-27], although these studies do not extensively link WAT expandability to immune cell infiltration and metabolic characterisation. This study is the first that focusses on all these processes simultaneously.

The size of adipocytes increased in all three WAT depots during body weight gain. Adipocyte number per fat pad remained comparable in all depots when correlated with body weight, whereas in gWAT and mWAT adipocyte numbers increased slightly during the HFD intervention when correlated with fat pad weight. Our observations are in line with previous mouse studies that showed expansion by hypertrophy only in sWAT, whereas mWAT expanded by both hypertrophy and hyperplasia $[25,27]$. Adipocytes in gWAT were larger than those in sWAT and mWAT in mice. Large adipocytes are thought to release more pro-inflammatory cytokines and chemokines that attract circulating monocytes into the WAT which, in turn, differentiate into macrophages $[5$, 28]. Indeed, the SVF of the gWAT contained a higher fraction of macrophages compared with sWAT and mWAT. Also, the absolute number of macrophages per WAT depot was higher in the gWAT than in the other WAT depots.

The absolute leucocyte numbers in mouse mWAT were much higher compared with sWAT and gWAT. The mWAT surrounds the intestine, which represents the first line of defence against intestinal pathogens and could therefore explain the large number of leucocytes. However, mWAT is also known to contain a large amount of lymphoid tissue including lymph nodes and milky spots [29]. Although we took great care to remove all visible lymph nodes from the mWAT before the immune cell characterisation, we cannot exclude the possibility that we missed some lymph nodes. As lymph nodes contain numerous leucocytes, this could also explain the high number of leucocytes present in mouse mWAT. Another issue affecting analyses of WAT inflammation might be contamination of the SVF with immune cells from the circulation. However, our mice were perfused before removal of the WAT depots.

Numerous pathophysiological processes are known to be associated with the development of IR. In this study, we focussed on WAT expendability and inflammation as a measure for WAT dysfunction. However, inadequate angiogenesis and related hypoxia are known as early determinants for WAT dysfunction as well $[30,31]$, and can induce WAT fibrosis, which has also been associated with IR [32]. Although beyond the scope of the current study, it is interesting to determine the association between these pathologies, WAT expansion and inflammation. Macrophages are highly abundant in WAT with, specifically, M1 macrophages accumulating during obesity and contributing to IR. Our data showed a phenotypic switch from M2 to M1 type macrophages during obesity, which has previously also been shown by Lumeng et al
[17]. The number of CLS, which are primarily formed by M1 macrophages, also increased with body weight [33]. M1 macrophages are known to accumulate lipids and form foam cells. Lipid accumulation in macrophages has previously been directly related to the expansion of WAT [34]. Whether lipidloaded macrophages are a consequence of the limited expansion of WAT remains to be investigated.

BAT is known to be a prominent player in body weight control, as it burns TG to produce heat $[35,36]$. Here, we show that BAT weight is strongly correlated with body weight. In general, high BAT weight is associated with inactive BAT, as TG is being stored instead of being used for heat production [37]. This is supported by an increased lipid droplet content in BAT with higher body weight. Our observed correlation between BAT weight and body weight can be explained by the thermal insulation function of WAT. The increased WAT in obesity is enough to keep the animal warm and heat production by BAT activity is reduced. Thus, body weight is an important confounder when studying BAT activity.

We conclude that mouse WAT depots vary considerably in expandability and immune cell composition during HFDinduced body weight gain. With a body weight threshold of approximately $40 \mathrm{~g}$ in mice, gWAT seems to have reached its maximum expansion capacity and at this point WAT dysfunction and concomitant systemic metabolic dysfunction will commence.

Acknowledgements The authors would like to thank T. C. M. Streefland (Department of Medicine, Division of Endocrinology, Leiden University Medical Center, the Netherlands) for excellent technical assistance.

Funding This work was supported by grants from the Center of Medical Systems Biology (CMSB), the Netherlands Consortium for Systems Biology (NCSB) established by the Netherlands Genomics Initiative/Netherlands Organization for Scientific Research (NGI/ NWO), the Leiden University Medical Center and Rembrandt Institute of Cardiovascular Science (RICS). PCNR is Established Investigator of the Netherlands Heart Foundation (grant 2009T038).

Duality of interest The authors declare that there is no duality of interest associated with this manuscript.

Contribution statement All authors made substantial contributions to the conception and design of the various aspects of the study or to the acquisition, analysis or interpretation of the data. All authors also contributed to drafting the article or revising it critically for important intellectual content and have given final approval of the version to be published. $\mathrm{VvH}$ is responsible for the integrity of this work as a whole.

Open Access This article is distributed under the terms of the Creative Commons Attribution 4.0 International License (http://creativecommons.org/licenses/by/4.0/), which permits unrestricted use, distribution, and reproduction in any medium, provided you give appropriate credit to the original author(s) and the source, provide a link to the Creative Commons license, and indicate if changes were made. 


\section{References}

1. Lee MJ, Wu Y, Fried SK (2013) Adipose tissue heterogeneity: implication of depot differences in adipose tissue for obesity complications. Mol Asp Med 34:1-11

2. Despres JP, Lemieux I (2006) Abdominal obesity and metabolic syndrome. Nature 444:881-887

3. Bjorndal B, Burri L, Staalesen V, Skorve J, Berge RK (2011) Different adipose depots: their role in the development of metabolic syndrome and mitochondrial response to hypolipidemic agents. J Obes 2011:490650

4. Tran TT, Yamamoto Y, Gesta S, Kahn CR (2008) Beneficial effects of subcutaneous fat transplantation on metabolism. Cell Metab 7: $410-420$

5. Xu H, Barnes GT, Yang Q et al (2003) Chronic inflammation in fat plays a crucial role in the development of obesity-related insulin resistance. J Clin Invest 112:1821-1830

6. Hotamisligil GS (2006) Inflammation and metabolic disorders. Nature 444:860-867

7. van Beek L, Lips MA, Visser A et al (2014) Increased systemic and adipose tissue inflammation differentiates obese women with T2DM from obese women with normal glucose tolerance. Metab Clin Exp 63:492-501

8. Murano I, Barbatelli G, Parisani V et al (2008) Dead adipocytes, detected as crown-like structures, are prevalent in visceral fat depots of genetically obese mice. J Lipid Res 49:1562-1568

9. Cinti S, Mitchell G, Barbatelli G et al (2005) Adipocyte death defines macrophage localization and function in adipose tissue of obese mice and humans. J Lipid Res 46:2347-2355

10. Winer DA, Winer S, Shen L et al (2011) B cells promote insulin resistance through modulation of $\mathrm{T}$ cells and production of pathogenic IgG antibodies. Nat Med 17:610-617

11. Wu H, Ghosh S, Perrard XD et al (2007) T cell accumulation and regulated on activation, normal $\mathrm{T}$ cell expressed and secreted upregulation in adipose tissue in obesity. Circulation 115:1029-1038

12. Hotamisligil GS, Shargill NS, Spiegelman BM (1993) Adipose expression of tumor necrosis factor-alpha: direct role in obesitylinked insulin resistance. Science 259:87-91

13. Shoelson SE, Lee J, Goldfine AB (2006) Inflammation and insulin resistance. J Clin Invest 116:1793-1801

14. Chau YY, Bandiera R, Serrels A et al (2014) Visceral and subcutaneous fat have different origins and evidence supports a mesothelial source. Nat Cell Biol 16:367-375

15. Vatier C, Kadiri S, Muscat A, Chapron C, Capeau J, Antoine B (2012) Visceral and subcutaneous adipose tissue from lean women respond differently to lipopolysaccharide-induced alteration of inflammation and glyceroneogenesis. Nutr Diabetes 2, e51

16. Bigornia SJ, Farb MG, Mott MM et al (2012) Relation of depotspecific adipose inflammation to insulin resistance in human obesity. Nutr Diabetes 2, e30

17. Lumeng CN, Bodzin JL, Saltiel AR (2007) Obesity induces a phenotypic switch in adipose tissue macrophage polarization. J Clin Invest 117:175-184

18. Vroegrijk IO, van Klinken JB, van Diepen JA et al (2013) CD36 is important for adipocyte recruitment and affects lipolysis. Obesity 21:2037-2045
19. Weisberg SP, McCann D, Desai M, Rosenbaum M, Leibel RL, Ferrante AW Jr (2003) Obesity is associated with macrophage accumulation in adipose tissue. J Clin Invest 112:1796-1808

20. Kintscher U, Hartge M, Hess K et al (2008) T-lymphocyte infiltration in visceral adipose tissue: a primary event in adipose tissue inflammation and the development of obesity-mediated insulin resistance. Arterioscler Thromb Vasc Biol 28:1304-1310

21. Nishimura S, Manabe I, Nagasaki M et al (2009) CD8+ effector T cells contribute to macrophage recruitment and adipose tissue inflammation in obesity. Nat Med 15:914-920

22. Sackmann-Sala L, Berryman DE, Munn RD, Lubbers ER, Kopchick JJ (2012) Heterogeneity among white adipose tissue depots in male C57BL/6J mice. Obesity 20:101-111

23. Marchesini G, Brizi M, Bianchi G et al (2001) Nonalcoholic fatty liver disease: a feature of the metabolic syndrome. Diabetes 50 : 1844-1850

24. Strissel KJ, Stancheva Z, Miyoshi H et al (2007) Adipocyte death, adipose tissue remodeling, and obesity complications. Diabetes 56 : 2910-2918

25. Wang QA, Tao C, Gupta RK, Scherer PE (2013) Tracking adipogenesis during white adipose tissue development, expansion and regeneration. Nat Med 19:1338-1344

26. Joe AW, Yi L, Even Y, Vogl AW, Rossi FM (2009) Depot-specific differences in adipogenic progenitor abundance and proliferative response to high-fat diet. Stem Cells 27:2563-2570

27. Jo J, Gavrilova O, Pack S et al (2009) Hypertrophy and/or hyperplasia: dynamics of adipose tissue growth. PLoS Comput Biol 5, e1000324

28. Skurk T, Alberti-Huber C, Herder C, Hauner H (2007) Relationship between adipocyte size and adipokine expression and secretion. $\mathrm{J}$ Clin Endocrinol Metab 92:1023-1033

29. Shimotsuma M, Shields JW, Simpson-Morgan MW et al (1993) Morpho-physiological function and role of omental milky spots as omentum-associated lymphoid tissue (OALT) in the peritoneal cavity. Lymphology 26:90-101

30. Hosogai N, Fukuhara A, Oshima K et al (2007) Adipose tissue hypoxia in obesity and its impact on adipocytokine dysregulation. Diabetes 56:901-911

31. Rausch ME, Weisberg S, Vardhana P, Tortoriello DV (2008) Obesity in C57BL/6J mice is characterized by adipose tissue hypoxia and cytotoxic $\mathrm{T}$ cell infiltration. Int $\mathrm{J}$ Obes 32:451-463

32. Halberg N, Khan T, Trujillo ME et al (2009) Hypoxia-inducible factor 1alpha induces fibrosis and insulin resistance in white adipose tissue. Mol Cell Biol 29:4467-4483

33. Lumeng CN, DelProposto JB, Westcott DJ, Saltiel AR (2008) Phenotypic switching of adipose tissue macrophages with obesity is generated by spatiotemporal differences in macrophage subtypes. Diabetes 57:3239-3246

34. Prieur X, Mok CY, Velagapudi VR et al (2011) Differential lipid partitioning between adipocytes and tissue macrophages modulates macrophage lipotoxicity and M2/M1 polarization in obese mice. Diabetes 60:797-809

35. Cannon B, Nedergaard J (2004) Brown adipose tissue: function and physiological significance. Physiol Rev 84:277-359

36. Harms M, Seale P (2013) Brown and beige fat: development, function and therapeutic potential. Nat Med 19:1252-1263

37. Townsend KL, Tseng YH (2014) Brown fat fuel utilization and thermogenesis. Trends Endocrinol Metab 25:168-177 